\title{
A comparative expression analysis of four MRX genes regulating intracellular signalling via small GTPases
}

\author{
Matthias Kohn ${ }^{1}$, Peter Steinbach ${ }^{1}$, Horst Hameister ${ }^{1}$ and Hildegard Kehrer-Sawatzki ${ }^{*}, 1$ \\ ${ }^{1}$ Department of Human Genetics, University of Ulm, Albert-Einstein-Allee 11, Ulm 89081, Germany
}

The $\mathrm{X}$ chromosomal mental retardation genes have attained high interest in the past. A rough classification distinguishes syndromal mental retardation (MRXS) and nonsyndromal mental retardation (MRX) conditions. The latter are suggested to be responsible for human specific development of cognitive abilities. These genes have been shown to be engaged in chromatin remodelling or in intracellular signalling. During this analysis, we have compared the expression pattern in the mouse of four genes from the latter class of MRX genes: Ophn1, Arhgef6 (also called $\alpha$ Pix), Pak3, and Gdi1. Ophn1, Pak3, and Gdi1 show a specific neuronal expression pattern with a certain overlap that allows to assign these signalling molecules to the same functional context. We noticed the highest expression of these genes in the dentate gyrus and cornu ammonis of the hippocampus, in structures engaged in learning and memory. A completely different expression pattern was observed for Arhgef6. In the CNS, it is expressed in ventricular zones, where neuronal progenitor cells are located. But Arhgef6 expression is also found in other nonneural tissues. Our analysis provides evidence that these signalling molecules are involved in different spatio-temporal expression domains of common signalling cascades and that for most tissues considerable functional redundancy of Rho-mediated signalling pathways exists.

European Journal of Human Genetics (2004) 12, 29-37. doi:10.1038/sj.ejhg.5201085

Published online 3 December 2003

Keywords: MRX; Arhgef6; Ophn1; Gdi1; Pak3; expression analysis

\section{Introduction}

In the last few years, much interest has been focused on the identification of $\mathrm{X}$ chromosomal genes which, when mutated, lead to mental retardation (for review see Chelly and Mandel ${ }^{1}$ ). Biannual workshops have been conducted to define new entities, which were added to the growing list of X-linked mental retardation syndromes. In the last update, 202 different entities had been listed. ${ }^{2}$ According to conventional use, a rough classification distinguishes between a majority of syndromal or specific mental retardation conditions (MRXS), and nonsyndromal or nonspecific mental retardation disorders (MRX). MRXS

${ }^{*}$ Correspondence: Dr H Kehrer-Sawatzki, Department of Human Genetics, University of Ulm, Albert-Einstein-Allee 11, Ulm 89081, Germany. Tel: + 49731 50023416; Fax: + 49731 50023438;

E-mail: hildegard.kehrer-sawatzki@medizin.uni-ulm.de

Received 15 April 2003; revised 24 June 2003; accepted 18 July 2003 are characterized by a specific pattern of neurological, physical, and/or metabolic abnormalities, whereas, in MRX, mental retardation is the only symptom. Therefore, the genes causing MRX are thought to be attractive candidate genes for human specific development of cognitive abilities. ${ }^{3}$ In the past, several reviews were addressed to these MRX genes. ${ }^{1,4,5,6}$ Chelly and Mandel ${ }^{1}$ introduced a new classification of the genes associated with mental retardation, which considers the functional aspects of the encoded proteins. According to this classification, a subgroup of MRX and also MRXS genes is engaged in chromatin remodelling, and by this in epigenetic modifications in neuronal cells. It is specific for this class of genes that some mutations cause MRX and other mutations cause phenotypically different entities of MRXS. Another class of MRX genes is engaged in intracellular signalling. These are authentic nonsyndromal mental retardation genes, since no phenotype other than mental 
retardation is observed in patients with mutations of these genes. Mental retardation in these patients is not caused by gross brain structural anomalies or by alterations of the thickness of cortical layers. ${ }^{7}$ MRX genes involved in intracellular signalling are: OPHN1, ARHGEF6, PAK3, and GDI1.

OPHN1 (oligophrenin) is a Rho-GTPase-activating protein, ${ }^{8}$ whereas ARHGEF6, also called $\alpha$ PIX, belongs to the group of guanine nucleotide exchange factors (GEF) for Rho-GTPases. ${ }^{9}$ PAK3 (p21 activated kinase 3) is a downstream effector of Rho GTPases, like Rac and Cdc $42 .{ }^{10}$ The GDP dissociation inhibitor GDI1 controls the activity of GTPases like Rab3a, by maintaining Rab3a in the inactive GDP-bound form. ${ }^{11,12}$

RhoA, Rac1, and Cdc42 are important mediators of extraand intracellular signals that regulate changes of the actin cytoskeleton (reviewed in $\mathrm{Luo}^{13}$ ). In neuronal cells, these changes go along with axonal growth and dendritic branching. Rab-GTPases are involved in vesicle formation and intracellular vesicular trafficking, and are essential in the regulation of synaptic connectivity in neurons. ${ }^{14}$ Therefore, small GTPases are integral signalling molecules engaged in the regulation of neuronal plasticity. The specific network of small GTPases determines the differentiation state of the respective neuronal cells. ${ }^{15}$ Neuronal cells are extremely vulnerable to the dysregulation of these signalling cascades, and tight constraints rest on the network of interacting signalling cascades in this cell type. This is reflected by the observation that mutations of the respective genes cause solely mental retardation, although these genes are active in various other tissues.

The MRX genes and their proteins are thought to be involved in the development of higher cognitive abilities. ${ }^{3,15}$ Therefore, profound knowledge about the specific neuronal structures expressing these MRX genes is of great importance. To identify these structures, we performed RNA in situ hybridization at different stages of mouse embryonic development and of the adult mouse brain. We also focused on whether there is a complete overlap of the spatio-temporal expression pattern of these genes. A third aim of the present study was to identify organs besides the CNS, which disclose distinct expression of these MRX genes.

\section{Material and methods Northern blot analysis}

A multiple-tissue Northern blot (MTN, Clontech, Palo Alto, CA, USA) of poly(A) + RNA from different tissues of adult mice was hybridized according to the manufacturer's protocol. As probes for Northern blot hybridizations, we used PCR products amplified with primers listed in Table 1. The PCR probes were amplified from IMAGE cDNA clones as templates, and spanned the end of the coding region and parts of the $3^{\prime} \mathrm{UTR}$ in the case of Pak3 and Arhgef6. The Gdi1 and Ophn1 probes are from the second half of the coding region (Table 1 ). The specificity of our RNA in situ hybridization probes is documented by sequence comparisons, as summarized in Table 2. These comparisons show that the homology of our probes to the sequence of functionally related genes is below $79 \%$ or even lower, so that crosshybridization can be excluded.

We chose the smaller PCR products as probes and not the full-length inserts of the IMAGE clones, since they produced less background on the filters. Probes were labelled by ${ }^{32}$ P-dCTP (Amersham Biosciences Europe) using Klenow polymerase and random hexamer oligonucleotides.

\section{Preparation of mouse embryos and adult mice}

Pregnant C57Bl/6 mice from overnight matings (the morning of vaginal plug detection was considered as the embryonic day (ED) 0.5) were killed by cervical dislocation. The embryos and other tissues were fixed overnight with $4 \%$ paraformaldehyde at $4{ }^{\circ} \mathrm{C}$, and were subsequently prepared for cryostat sectioning as described. ${ }^{16}$ Serial cryosections of $10 \mu \mathrm{m}$ were mounted on slides.

Table 1 IMAGE clones and primers to amplify probes for Northern blot hybridization

\begin{tabular}{|c|c|c|c|c|c|}
\hline MRX gene & Primers $5^{\prime} \rightarrow 3^{\prime}$ & Probe size $(b p)$ & $\begin{array}{c}\text { IMAGE clone ID } \\
\text { (GenBank accession } \\
\text { number) }\end{array}$ & $\begin{array}{l}\text { PCR probe position on } \\
\text { CDNA (acc. no.) }\end{array}$ & $\begin{array}{l}\text { Position of IMAGE clone } \\
\text { insert with respect to } \\
\text { cDNA (acc. no.) }\end{array}$ \\
\hline Arhgef6 & $\begin{array}{l}\text { AGTACTGCTGCCСTGGAAGA } \\
\text { CCACAAAGCACAAAGCTCAA }\end{array}$ & 467 & $\begin{array}{c}3994508 \\
(B F 167398)\end{array}$ & $\begin{array}{c}2169-2636 \\
\left(N M \_152081\right)\end{array}$ & $\begin{array}{c}2069-4567 \\
\left(N_{1}+152801\right)\end{array}$ \\
\hline Gdi1 & $\begin{array}{l}\text { TGACCTGGGTCAAGATGTCA } \\
\text { ATGCAGGCTCAACTTCCTTTT }\end{array}$ & 583 & $\begin{array}{c}1068062 \\
(A A 764456)\end{array}$ & $\begin{array}{c}910-1493 \\
\left(X_{-} 135916\right)\end{array}$ & $\begin{array}{c}851-1650 \\
\left(X_{-} 135916\right)\end{array}$ \\
\hline Pak3 & $\begin{array}{l}\text { AAACGAAGCACTATGGTGGG } \\
\text { TGGCTACTTCGACATTGCTG }\end{array}$ & 608 & $\begin{array}{c}761330 \\
(\mathrm{AA} 388261)\end{array}$ & $\begin{array}{c}1768-2376 \\
\left(N M \_008778\right)\end{array}$ & $\begin{array}{c}1768-2376 \\
(\text { NM_008778) }\end{array}$ \\
\hline Ophn1 & $\begin{array}{l}\text { AATGGCTCACCACAAGGAAG } \\
\text { CTGAACCСТACAAGGCCAGA }\end{array}$ & 417 & $\begin{array}{c}2812668 \\
(\mathrm{AW} 412751)\end{array}$ & $\begin{array}{c}2362-2753 \\
\left(N M \_052976\right)\end{array}$ & $\begin{array}{c}1657-3040 \\
(\text { NM_002547) }\end{array}$ \\
\hline
\end{tabular}


Table 2 Sequence comparisons of IMAGE clones used for RNA in situ hybridization with functionally related genes

\begin{tabular}{|c|c|c|c|}
\hline \multirow[t]{2}{*}{$\begin{array}{l}\text { Gene name (accession number of } \\
\text { IMAGE clone used for RISH) }\end{array}$} & \multirow[t]{2}{*}{ Related genes (accession number) } & \multicolumn{2}{|c|}{ Sequence identity (\%) as determined by } \\
\hline & & Blast analysis & Gap program \\
\hline $\begin{array}{l}\text { Pak3 } \\
\text { (AA388261) }\end{array}$ & $\begin{array}{l}\text { Pak1 (NM011035) } \\
\text { Pak2 (BC029195) } \\
\text { Pak4 (NM027470) }\end{array}$ & $\begin{array}{l}79.0 \\
77.0 \\
79.0\end{array}$ & $\begin{array}{l}78.0 \\
77.5 \\
55.8\end{array}$ \\
\hline Ophn1 (AW412751) & - & - & - \\
\hline
\end{tabular}

- , No identity detected.

\section{RNA in situ hybridization}

RNA in situ hybridization was performed with antisense and sense riboprobes generated by in vitro transcription with T3, T7 or Sp6 polymerases in the presence of $\alpha^{35}$ S-UTP (Amersham Biosciences Europe) from linearized DNA of IMAGE cDNA clones. The sequences used as RNA probes corresponded to the end of the coding region and the 3'UTR of Pak3 and Arhgef6. The Gdi1 and Ophn1 probes span the second half of the coding region (Table 1). Hybridizations were carried out overnight as previously described. ${ }^{16}$

\section{Results}

Expression of Arhgef6, Gdi1, Ophn1, and Pak3 in adult mouse tissues

To compare the expression pattern of these MRX genes in different adult tissues, we performed Northern blot hybridizations. A $4.4 \mathrm{~kb}$ transcript of Arhgef6 was identified, which is prominently expressed in the heart and at lower levels in all the tissues analysed, including the total brain. This $4.4 \mathrm{~kb}$ isoform of Arhgef6 has also been observed by Kutsche and $\mathrm{Gal}^{17}$ and Manser et al. ${ }^{18}$ Additionally, in testis, transcripts of 3.6 and $2.2 \mathrm{~kb}$ (more prominent) were noticed (Figure 1a). Most probably, these isoforms result from the usage of alternate polyadenylation sites. Ophn1 is also highly expressed in the testis as a transcript of 4.4 and $2.4 \mathrm{~kb}$ (less prominent). Low levels of an $\sim 7 \mathrm{~kb}$ Ophn1 transcript described by Billuart et $a l^{8}$ are observed in the brain and heart (Figure 1b). The strongest expression of a $2.6 \mathrm{~kb}$ Gdi1 transcript is found in the kidney, liver, brain, and heart. Weaker expression of this transcript is observed in the testis and lung. A second Gdi1 transcript of $\sim 3 \mathrm{~kb}$ is found to be moderately expressed in the kidney, liver, lung, brain, and heart (Figure 1c). Three isoforms of Pak3 were observed, a testis-specific transcript of $2.2 \mathrm{~kb}$, a transcript of
$2.4 \mathrm{~kb}$ expressed in the brain and testis, and a Pak3 isoform of $\sim 3 \mathrm{~kb}$ in the kidney and brain (Figure $1 \mathrm{~d}$ ). The $\sim 3 \mathrm{~kb}$ isoform has been described by Burbelo et al. ${ }^{19}$ The 2.2 and 2.4 Pak3 transcripts result from the usage of alternate polyadenylation sites and alternative splicing in the $5^{\prime} \mathrm{UTR}$, as documented by the structure of the cDNA clones with the accession numbers BM932922 and BY257719. The strongest expression of Arhgef6, Ophn1, and Pak3 is found in the adult testis, whereas the highest Gdi1 expression is noticed in the brain.

Spatio-temporal expression pattern of Arhgef6, Gdi1, Ophn1, and Pak3 during embryonic development To evaluate the expression pattern of these MRX genes during development, we performed RNA in situ hybridization on sections of mouse embryos. At early midgestational stages, ED 9.5-10.5, Ophn1, Pak3, and Gdi1 are not specifically expressed in the embryo proper, in which the neural tube is the most prominent organ at this time. These genes are also not expressed in the placenta adhering to the embryo. Arhgef6, however, is highly expressed in the foetal parts of the placenta, but not in the embryo (not shown).

Beginning from ED 11.5, a distinct expression pattern confined to the CNS is observed for Gdi1, and with reduced intensity for Pak3 and Ophn1. Upon closer examination, expression differences between the genes become apparent. Pak3 shows its highest expression at the base of the diencephalon in the supraoptic area, which, however, is spared by Gdi1 expression. High levels of Ophn1 transcripts are noticed in the striatum and the epithalamus, which were spared by Gdi1 and Pak3. For all the three genes, the mRNAs are enriched in the spinal cord, medulla oblongata, and the hypothalamus (Figure $2 \mathrm{~b}-\mathrm{d}$ ).

In contrast to Ophn1, Gdi1, and Pak3, a highly specific and sharply confined expression signal of Argef6 is noticed at ED 12.5 in the ventricular zone of the myelon- and 

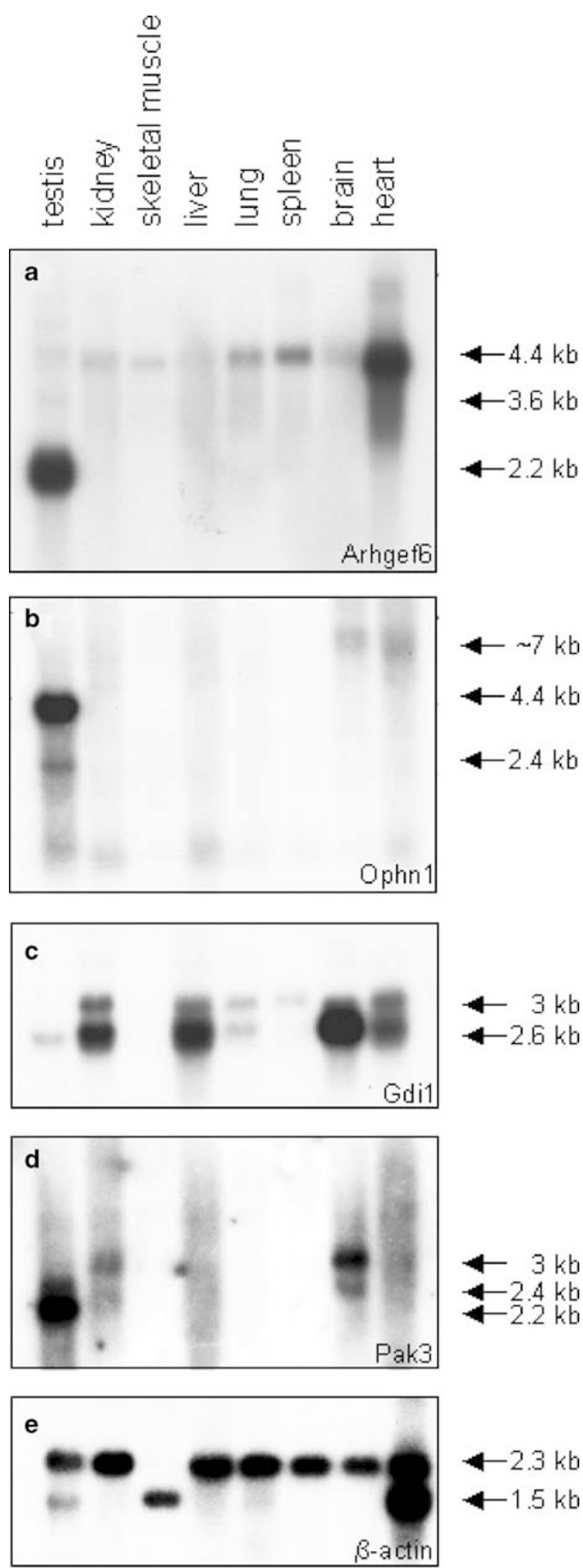

Figure 1 Northern blot analysis of Arhgef6 (a), Ophn1 (b), Gdi1 (c), Pak3 (d), and $\beta$-actin (e). metenencephalon, which borders the IVth ventricle, and in the ganglionic eminence protruding in the lateral ventricles (Figure 2e). The high expression of Argef6 in the ventricular zone persists up to ED 14.5 (Figure $2 \mathrm{j}$ ). In Figure $2 \mathrm{e}$, some extraplacental membranes are attached to the embryo, which show high Arhgef6 activity.

At a later stage (ED 14.5), Ophn1, Pak3, and Gdi1 show a similar pattern as they are only expressed in structures of the CNS (Figure 2g-i). Again, there are slight, but clear, spatial differences in their expression pattern. Pak3 is highly expressed in the supraoptic area and striae medullaris. These regions, however, are spared again by Gdi1 expression. At this developmental stage, the neocortex of the telencephalon is separated into a subventricular and an intermediate zone. Gdi1 and also Ophn1 are expressed in the intermediate zone, whereas Pak3 mRNA is expressed more in the subventricular zone. Only Gdi1 and Ophn1 transcripts are observed in the pituitary gland, the peripheral cranial and dorsal root ganglia, and in the olfactory epithelium. Enhanced Pak3 expression is not noticed in these structures. Arhgef6 is highly expressed in specific areas of the brain, such as the ventricular layer of the ganglionic eminences lining the lateral ventricles and in the same layers of the metencephalon bordering the IVth ventricle (Figure $2 \mathrm{j}$ ). The specific expression of Arhgef6 is also observed in other organs outside of the brain such as the tongue, heart, and the epithelia of the bladder, and the allantoic remains in the umbilical cord.

At foetal stage ED 16.5, the expression pattern of Pak3, Ophn1, and Gdi1 remains confined to neural structures. Pak3 expression conforms now to those of Gdi1 and Ophn1, as they are all expressed in the cortical plate (data not shown). At this stage, continued radial migration of the neuronal cells in the neocortex is shaping the cortical plate, which forms at the outer (peripheral) site of the intermediate zone. ${ }^{20}$ Conspicuously, all the three genes are expressed in the cortical plate, which differentiates into the neocortical layers I-VI during postnatal life. At ED 16.5, Arhgef6 expression in the ventricular zones is already reduced (data not shown). It is interesting to note that Gdi1 expression is upregulated after birth in the kidney, liver, and heart (Figure 1c), since, in foetal stages, Gdi1 is not prominently expressed in these organs.

\section{Expression of Arhgef6, Gdi1, Ophn1, and Pak3 in the postnatal brain}

To investigate the expression of these MRX genes at postnatal stages, coronal sections of the complete head of 2-day-old mice were analysed (Figure 2k-o). Coronal sections are insofar informative as the large diencephalon consisting of the thalamus and hypothalamus and the inner archicortex, made up by the hippocampus, as well as the neocortex are visible. The most differentiated expression pattern is observed for Gdi1 (Figure 2n). Strong Gdi1 expression marks the dentate gyrus and the cornu 

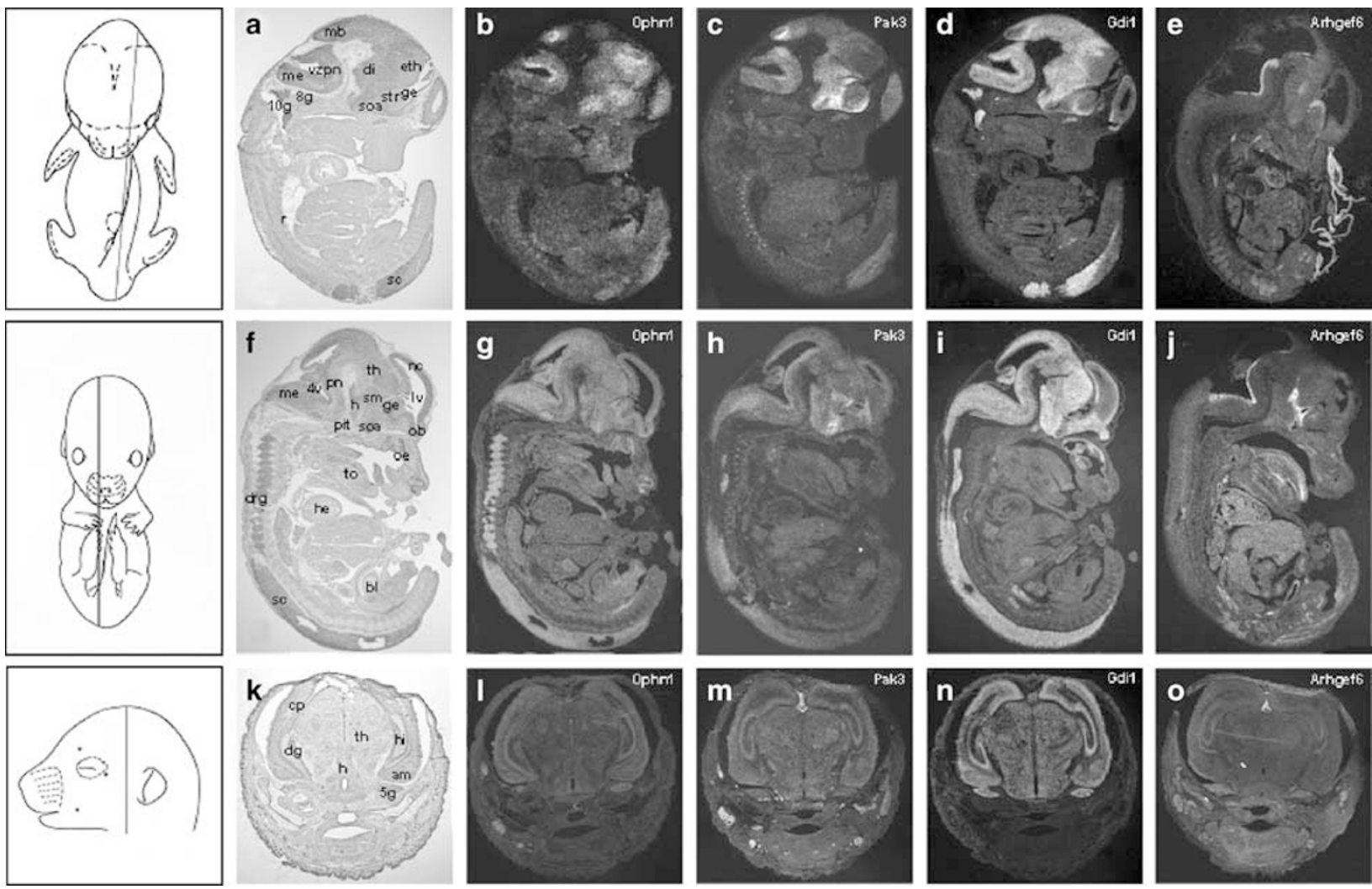

Figure 2 Ophn1 (b,g, l), Pak3 (c,h, m), Gdi1 (d,i, n), and Arhgef6 (e,j,o) expression on sagittal sections of ED 12.5 (a-e) and at ED $14.5(\mathbf{f}-\mathbf{j})$ mouse embryos and coronal sections of the head ( $\mathbf{k}-\mathbf{o})$ of a (2-day) new-born mouse. $4 v$, fourth ventricle; $5 g$, trigeminal ganglion; am, amygdaloid nucleus; bl, bladder; $d g$, dentate gyrus; di, diencephalon; drg, dorsal root ganglion; eth, epithalamus; $m b$, roof of the midbrain; ge, ganglionic eminence; $h$, hypothalamus; he, heart; hi, hippocampus; $l$, lateral ventricle; me, medulla oblongata; $m t$, metencephalon; $n c$, neocortex; ob, olfactory bulb; oe, olfactory epithelium; pit, pituitary gland; r, ribs; sc, spinal cord; str, striatum; sm, striae medullaris; soa, supraoptic area; th, thalamus; to, tongue; vzmt, ventricular zone of the metencephalon. Towards the left, the direction of the section is indicated in a scheme.

ammonis in all parts (CA1-CA3). The distribution of the signals in this section illustrates the continuation of the cornu ammonis from the archicortex to the cortical plate in the neocortex. Both tissues are marked by high Gdi1 expression. In the ventral part, Gdi1 is prominently expressed in the corpora amygdala and the dentate gyrus. This pattern is very similar to that observed for Ophn1 and Pak3 (Figure 2l, m), although their expression is less intensive. Arhgef6, however, is not specifically expressed in these structures (Figure 2o). A rather faint Arhgef6 signal, however, is noticed in the ventricular zone lining the cornu temporale of the lateral ventricle.

\section{Arhgef6, Gdi1, Ophn1, and Pak3 expression in the adult brain}

In coronal sections through the more anterior region of the adult brain (Figure 3a, c, e, g, i), distinctive Gdi1 expression is detected in the piriform cortex, thalamus, hypothalamus, the habenular nuclei, the whole cornu ammonis, and the dentate gyrus of the hippocampus. This pattern is similar, although more faint, to that observed for Ophn1 and Pak3.

The pattern of Arhgef6 is different (Figure 3i). The expression of Arhgef6 is enhanced in the peripheral CA3 part of the cornu ammonis and in the striae of the forceps major of the corpus callosum, which line the hippocampus on the outside. For none of the genes, a distinct signal in the mature neocortex is observed.

In sections of the posterior part of the adult brain (Figure $3 \mathrm{~b}, \mathrm{~d}, \mathrm{f}, \mathrm{h}, \mathrm{j}$ ), the cerebellum and the medulla oblongata are evaluated. The highest Gdi1 mRNA levels are detected in the Purkinje cells of the cerebellum (Figure 3h). Ophn1 expression is also noticed in these cells, although less distinct compared to Gdi1 (Figure 3d). In the granular layer of the cerebellar gyri, the mRNA levels of Gdi1 and Ophn1 are enhanced. The expression pattern of Arhgef6 contrasts to the pattern of the aforementioned genes. A faint signal marks the white matter in the pedunculi cerebelli and in the cerebellar gyri (Figure $3 \mathrm{j}$ ). In the medulla oblongata, Gdi1 is expressed in the nuclei cochlearis, vestibularis, and 

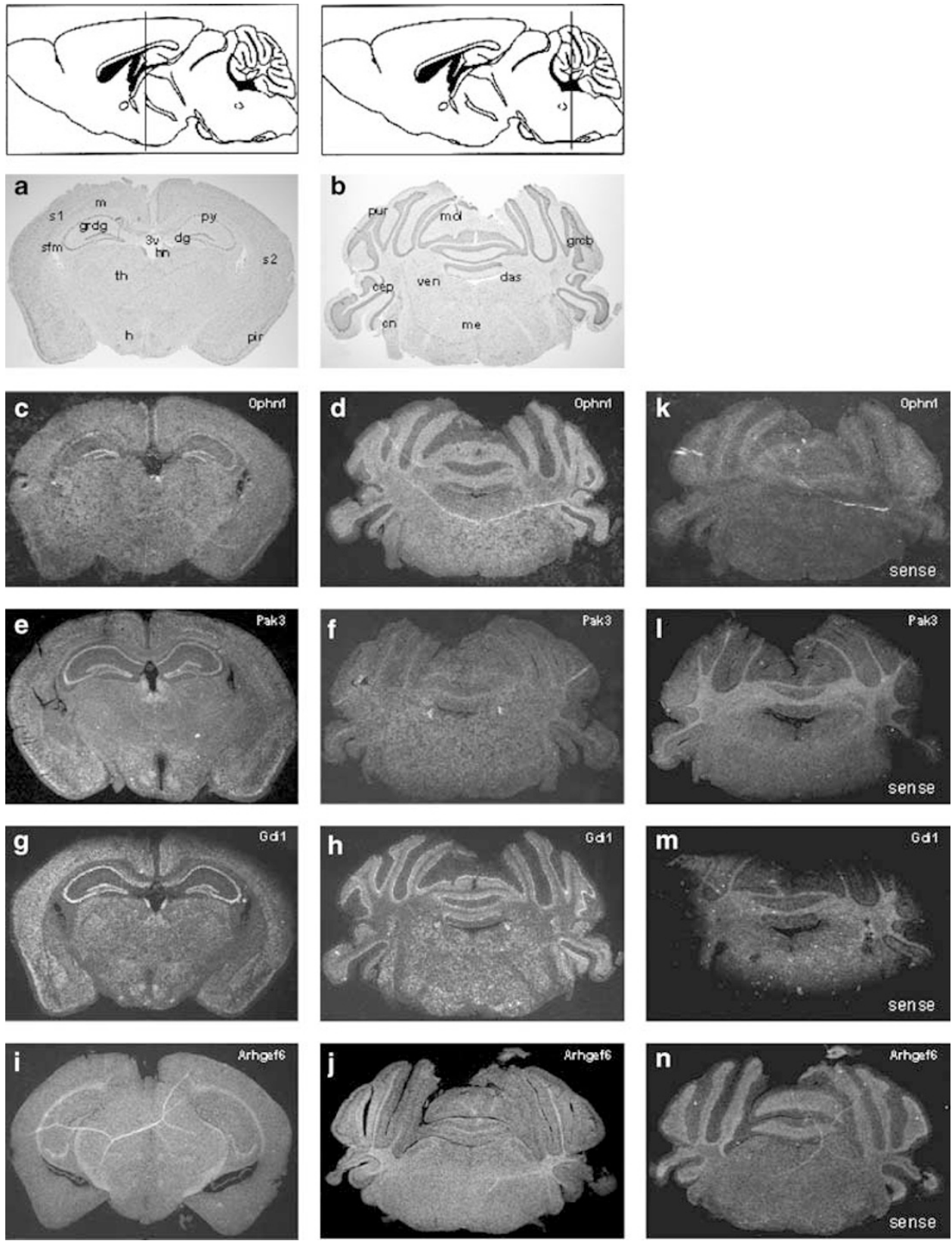

Figure 3 Ophn1 (c, d), Pak3 (e, f), Gdi1 ( $\mathbf{g}, \mathbf{h})$, and Arhgef6 (i, j) expression in the adult brain. Consecutive coronal sections from caudal to rostral have been used. The direction of the section is indicated in the scheme on the left. cn, cochlear nucleus; cep, cerebellar peduncle; $3 v$, dorsal third ventricle; das, dorsal acoustic striae; $d g$, dentate gyrus; grcb, granular layer of cerebellum; grdg, granular layer of dentate gyrus; $h$, hypothalamus; $h n$, medial habenular nuclei; $m$, motor cortex; me, medulla oblongata; mol, molecular layer; pir, piriform cortex; pur, Purkinje cells; py, pyramidal cell layer of hippocampus; s1, primary somatosensory cortex; s2, secondary somatosensory cortex; sfm, striae forceps major of the corpus callosum; th, thalamus; ven, vestibular nucleus. In $(\mathbf{k}, \mathbf{I}, \mathbf{m}, \mathbf{n})$, the hybridizations with the respective sense probes are indicated.

the dorsal acustic striae (Figure $3 \mathrm{~h}$ ). The other genes are not specifically expressed in the medulla oblongata.

Since Northern blot hybridizations (Figure 1) revealed a high expression of Arhgef6, Ophn1, and Pak3 in testis, we also investigated their expression pattern by in situ hybridization in sections of the mature testis (Figure 4). Spermatogenesis in rodent testis follows the described gametogenic cycle of germ cell proliferation. ${ }^{21}$ Therefore, the section through each tubulus seminiferus is specific for a certain stage during germ cell proliferation. Ophn1 is 

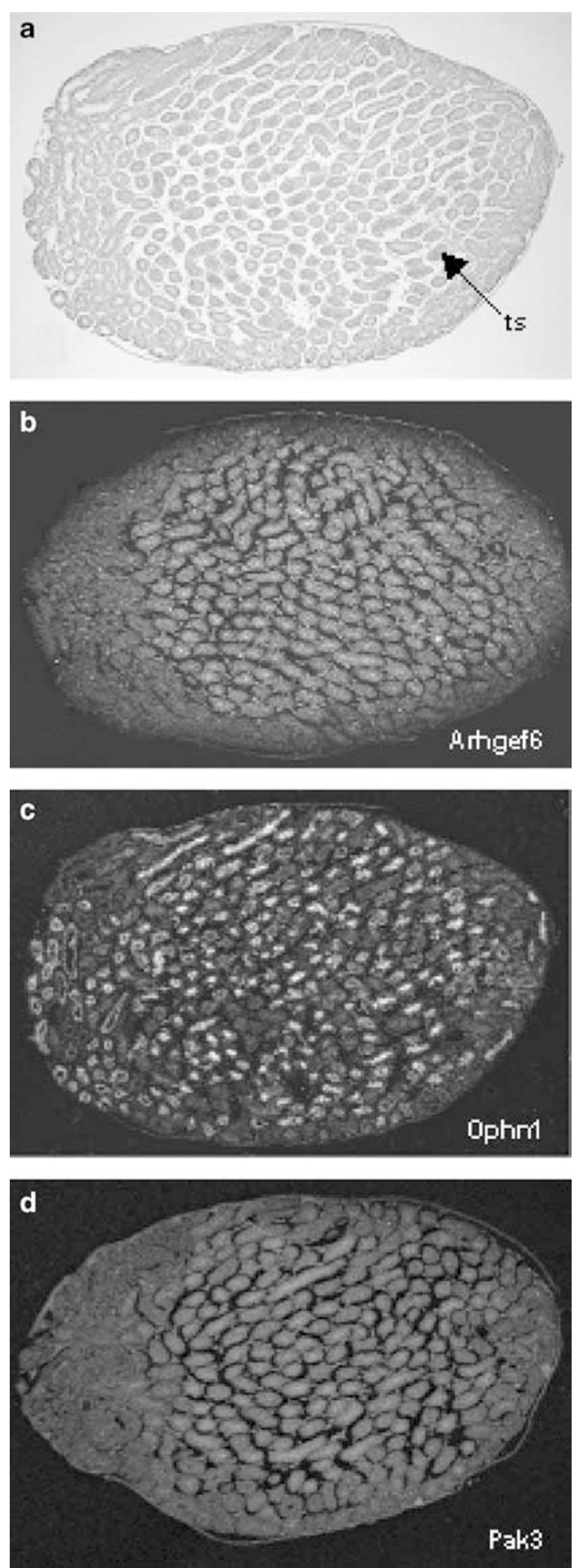

Figure 4 Arhgef6 (b), Ophn1 (c), and Pak3 (d) expression in sagittal sections from the adult testis. In the brightfield section (a), one tubulus (ts) is indicated by an arrow. An enhanced expression of Ophn1 (c) in stage IX-XII tubuli seminiferi is noticed. Arhgef6 (b) and Pak3 (d) are expressed in all tubuli seminiferi. expressed at a low level in every stage (Figure 4c). Enhanced expression is observed in the inner lumen of stage IX-XII tubuli seminiferi, where the morphological differentiation from spermatid to mature sperm takes place. This process occurs without cell division, and is one of the most phenomenal morphological differentiation steps. Arhgef6 and Pak3 show no specific expression pattern in the testis (Figure 4b, d) and Gdi1 is not expressed at all.

\section{Discussion}

The MRX genes Arhgef6, Ophn1, and Pak3 were chosen for a comparative expression analysis, as they interfere with the metabolism of the small GTPases RhoA, Rac1, and Cdc42. These GTPases act as molecular switches to mediate extraand intracellular stimuli necessary for changes of the cytoskeleton organization during cytokinesis, cellular migration, and cell shape remodelling during terminal differentiation (reviewed in Ramakers ${ }^{15}$ ). Migration is an integral process of neuronal cells, which originate from the ventricular zone and migrate radially to the surface of the cortex. In some instances, they even show tangential migration into another brain compartment. ${ }^{20}$ Evidently, cytokinesis, which is correlated with cell proliferation, and migration of neuronal cells are not impaired in MRX patients as no abnormal cortical lamination defects have been reported. ${ }^{7}$ There are no developmental but functional deficits of neurons in MRX patients. Dendritic spine abnormalities have been described in patients with mental retardation. $^{22,23}$ The organization of neurons is very complex. During terminal differentiation of neurons, an axon with enormous arborization in the target tissue and a large dendritic tree for afferent signal reception are formed. The dendritic tree is covered with up to $10^{4}$ spines, which are small actin-rich protrusions and form synapses with axons from other neurons. Recently, evidence was provided that not only are synapses in a state of permanent plasticity but also the spines themselves. ${ }^{24,25}$ Upon stimulation, reorganization of spines has been observed in the hippocampus and neocortex of the adult. This emphasizes the importance of the network, which regulates the cytoskeleton as well as vesicle trafficking in neuronal cells. Cdc42 and Rac1 have been shown to be positive regulators of neurite outgrowth, whereas RhoA inhibits neurite extension. ${ }^{26,17}$ Therefore, it is fascinating to realize that just these regulators are mutated in patients suffering from nonsyndromic mental retardation, MRX.

In vitro studies have proven a direct interaction of Arhgef6, Ophn1, and Pak3 with Rho GTPases. Ophn1 acts as a Rho GTPase-activating enzyme, ${ }^{8}$ Arhgef6 is a guanine exchange factor for Rac1 and Cdc42, and shows interaction with Pak proteins. ${ }^{9,18}$ Pak3 acts downstream from Rac1 and Cdc42 directly on the actin cytoskeleton and on gene 
expression. ${ }^{27}$ The assignment of Gdi1 to the signal transduction process of neuronal cells is more indirect, as Gdi1 is engaged in intracellular vesicle and synapse formation via other small GTPases, Rab3a and Rab3c. ${ }^{11,12}$ All these molecules play an integral part during signalling in and between neuronal cells. The aim of this study was to identify the structures expressing the MRX genes Arhgef6, Ophn1, Pak3, and Gdi1, and to compare their spatiotemporal expression pattern during development and in the adult brain.

Already by Northern blot analysis of different adult mouse tissues, considerable expression differences are apparent (Figure 1). In the adult brain, only Gdi1 and Pak3 are prominently expressed, as was shown previously. ${ }^{10,28}$ The relatively high expression levels of Arhgef6, Pak3, and Ophn1 in the testis are remarkable (Figure 1). Since genes relevant for human speciation show a tendency to be expressed simultaneously in the brain and testis, ${ }^{29}$ this suggests that MRX genes serve this function.

The in situ expression analysis of these genes shows a relative similarity of the pattern between Gdi1, Ophn1, and Pak3. These genes are specifically and exclusively expressed in neural tissues from ED 11.5 onwards. Although the expression patterns of Ophn1, Pak3, and Gdi1 are partly overlapping, regional differences of expression during development were observed (Figure $2 \mathrm{a}-\mathrm{j}$ ). In the adult brain, the highest expression of these genes is noticed in the dentate gyrus and cornu ammonis of the hippocampus in the archicortex and minor expression in the piriform cortex (Figure 3c-i). In human, the hippocampus is engaged in learning and memory, and is the dominant structure for activity-dependent synaptic plasticity in the mammalian brain. ${ }^{30}$ External stimuli are represented in the brain as spatio-temporal patterns of neuronal activity, that is, synaptic transmission. The exceptional importance of the hippocampal region is illustrated by its complex gene activity state. The overlapping and specific expression pattern of the MRX genes in the hippocampus underscores the importance of these genes in processes associated with learning and memory and the development of higher cognitive abilities.

Whereas the expression patterns of Ophn1, Pak3, and Gdi1 are similar and overlapping, Arhgef6 has a completely different pattern than that observed for the other MRX genes. Well-defined Arhgef6 signals are noticed in a thin layer of ventricular cells of the lateral and IVth ventricles (Figure 2e, j). This highly specific expression is present only during a small window of development from ED 12.5 to 14.5. At this stage, the neural cells in the ventricular regions show high proliferative activity. The distinctive and high expression of Arhgef6 at these sites suggests that Arhgef6 plays an important role in neuroepithelial proliferation. During embryonic development, Arhgef6 expression is not only restricted to the CNS, but also observed in the muscles of the tongue and heart, in the epithelia of the bladder (Figure 2j), and in the placental membranes (Figure 2e).

The comparison of the expression pattern of these genes for signalling molecules shows that there is a regionspecific variation of activity. Especially, Arhgef6 exemplifies a unique expression pattern, which indicates that Arhgef6 is not active in the same signalling cascade as the other investigated MRX genes are. ${ }^{1,13}$ In this context, the considerable functional redundancy of Rho-mediated signalling pathways has to be taken into account. There are at least 11 members of the Rho family, of which RhoA, Rac1, and Cdc42 have been most extensively studied in the past. Further, there are about 30 guanine nucleotide exchange factors, GEFs, and 13 GTPase-activating proteins, GAPs, supplemented by several GDP dissociation inhibitors (GDIs). The signalling cascades regulated by these molecules build up a highly complex network. This is seemingly robust enough to compensate for the absence of one factor, like one of the MRX gene products, in all tissues throughout development. However, in the most developed and highly active hippocampal neuronal cells, the functional constraints are so tight that disturbances in these networks lead to underperformance. The most universal outcome of hippocampal underperformance is nonsyndromic mental retardation.

\section{Acknowledgements}

We thank Dr Prof Ingrid Reisert, Ulm, for critical reading of the manuscript and Stefanie Fromm for technical assistance. This research was supported by a grant from the Deutsche Forschungsgemeinschaft (HA-1082/16).

\section{References}

1 Chelly M, Mandel JL: Monogenic causes of X-linked mental retardation. Nat Rev Genet 2001; 2: 669-680.

2 Chiurazzi P, Hamel BC, Neri G: XLMR genes: update 2000. Eur J Hum Genet 2001; 9: 71-81.

3 Zechner U, Wilda M, Kehrer-Sawatzki H, Vogel W, Fundele R, Hameister $\mathrm{H}$ : A high density of X-linked genes for general cognitive ability: a run-away process shaping human evolution? Trends Genet 2001; 17: 697-701.

4 Chelly J: Breakthroughs in molecular and cellular mechanisms underlying X-linked mental retardation. Hum Mol Genet 1999; 8: $1833-1838$.

5 Toniolo D, D'Adamo P: X-linked non-specific mental retardation. Curr Opin Genet Dev 2000; 10: 280-285.

6 Gécz J, Mulley J: Genes for cognitive function: developments on the X. Genome Res 2000; 10: 157-163.

7 Lubs H, Chiurazzi P, Arena J, Schwartz C, Tranebjaerg L, Neri G: XLMR genes: update 1998. Am J Med Genet 1999; 83: 237-247.

8 Billuart P, Bienvenu T, Ronce N et al Oligophrenin-1 encodes a rhoGAP protein involved in X-linked mental retardation. Nature 1998; 392: 923-926.

9 Kutsche K, Yntema H, Brandt A et al Mutations in ARHGEF6, encoding a guanine nucleotide exchange factor for Rho GTPases, in patients with X-linked mental retardation. Nat Genet 2000; 26: 247-250.

10 Allen KM, Gleeson JG, Bagrodia S et al PAK3 mutation in nonsyndromic X-linked mental retardation. Nat Genet 1998; 20: $25-30$. 
11 D'Adamo P, Menegon A, Lo Nigro C et al Mutations in GDI1 are responsible for X-linked non-specific mental retardation. Nat Genet 1998; 19: 134-139.

12 Bienvenu T, des Portes V, Saint Martin A et al Non-specific Xlinked semidominant mental retardation by mutations in a Rab GDP-dissociation inhibitor. Hum Mol Genet 1998; 7: 1311-1315.

13 Luo L: Rho GTPases in neuronal morphogenesis. Nat Rev Neurosci 2000; 1: 173-180.

14 Ishizaki H, Miyoshi J, Kamiya H et al Role of Rab GDP dissociation inhibitor $\alpha$ in regulating plasticity of hippocampal neurotransmission. Proc Natl Acad Sci USA 2000; 97: 1587-1592.

15 Ramakers GJA: Rho proteins, mental retardation and the cellular basis of cognition. Trends Neurosci 2002; 25: 191-199.

16 Schmid P, Schulz WA, Hameister H: Dynamic expression pattern of the myc protooncogene in midgestation mouse embryos. Science 1989; 243: 226-229.

17 Kutsche K, Gal A: The mouse Arhgef6 gene: cDNA sequence, expression analysis, and chromosome assignment. Cytogenet Cell Genet 2001; 95: 196-201.

18 Manser E, Loo TH, Koh CG et al PAK kinases are directly coupled to the PIX family of nucleotide exchange factors. Mol Cell 1998; 1: $183-192$.

19 Burbelo PD, Kozak CA, Finegold AA, Hall A, Pirone DM: Cloning, central nervous system expression and chromosomal mapping of the mouse PAK-1 and PAK-3 genes. Gene 1999; 232: 209-215.

20 Park HT, Wu J, Rao Y: Molecular control of neuronal migration. BioEssays 2002; 24: 821-827.

21 Oakberg EF: A description of spermiogenesis in the mouse and its use in analysis of the cycle of the seminiferous epithelium and germ cell renewal. Am J Anat 1956; 99: 391-414.
22 Purpura DP: Dendritic spine 'dysgenesis' and mental retardation. Science 1974; 186: 1126-1128.

23 Kaufmann WE, Moser HW: Dendritic anomalies in disorder associated with mental retardation. Cereb Cortex 2000; 10: 981-991.

24 Trachtenberg JT, Chen BE, Knott GW et al Long-term in vivo imaging of experience-dependent synaptic plasticity in adult cortex. Nature 2002; 420: 788-794.

25 Grutzendler J, Kasthuri N, Wen-Biao G: Long-term dendritic spine stability in the adult cortex. Nature 2002; 420: 812-816.

26 Tashiro A, Minden A, Yuste R: Regulation of dendritic s pine morphology by the rho family of small GTPases: antagonistic roles of Rac and Rho. Cereb Cortex 2000; 10: 927-938.

27 Daniels RH, Bokoch GM: p21-activated protein kinase: a crucial component of morphological signalling? Trends Biochem Sci 1999; 24: $350-355$

28 Baechner D, Sedlacek Z, Korn B, Hameister H, Poustka A Expression patterns of two human genes coding for different rab GDP-dissociation inhibitors (GDIs), extremely conserved proteins involved in cellular transport. Hum Mol Genet 1995; 4: $701-708$.

29 Wilda M, Baechner D, Zechner U, Kehrer-Sawatzki H, Vogel W, Hameister H: Do the constraints of human speciation cause expression of the same set of genes in brain, testis, and placenta? Cytogenet Cell Genet 2001; 91: 300-302.

30 Bliss TVP, Collingridge GL: A synaptic model of memory: long-term potentiation in the hippocampus. Nature 1993; 361: $31-39$. 